\title{
Erosion de cavitation dans une turbine Pelton : essais de marquage et application d'une méthode de prévision
}

\author{
par A. Archer*, J.-M. Dorey*, G. Rossi**, R. Gambacciani*** \\ Electricité de France* - DER, Riva Hydroart**, ENEL***
}

\section{I — INTRODUCTION}

Le contrat de recherche européen BRITE-EURAM sur la "Prévision de l'érosion de cavitation dans les machines hydrauliques " s'est déroulé de 1992 à 1995. Ce contrat de recherche a rassemblé huit partenaires : des exploitants de centrales hydroélectriques (EDF - France, ENEL - Italie), des constructeurs de pompes (KSB - Allemagne), de turbines (Riva-Hydroat - Italie), d'hélices (Cehipar - Espagne), et des laboratoires de recherche industriels (ACB-Cerg - France, coordinateur du projet) et universitaires (DTU: Université Technologique de Darmstadt - Allemagne, et EPFL : Ecole Polytechnique Fédérale de Lausanne - Suisse). Ses objectifs étaient de définir les paramètres caractéristiques des écoulements cavitants érosifs et d'établir des lois de transposition en échelle, vitesse et matériau, afin de prédire l'érosion de cavitation sur machines industrielles (pompe, hélice, turbine Francis et turbine Pelton) à partir d'essais sur modèles.

Une turbine Pelton peut être soumise à différents types d'usure : une érosion par abrasion de particules solides véhiculées dans le fluide et impactant à grande vitesse les augets, une érosion par impact de gouttelettes et une érosion par cavitation [1][2]. Cet article décrit des essais de marquage par cavitation, consistant à enregistrer les marques des implosions des structures de vapeur sur une surface métallique polie, sur une turbine Pelton prototype et sur son modèle et présente l'application d'une méthode de prévision de l'érosion.

\section{II $\square$ ESSAIS SUR LA MACHINE PROTOTYPE}

\section{- 2.1. Caractéristiques de la machine}

La turbine Pelton de Ceto-Palobbia (Italie) présente une forte érosion par cavitation nécessitant des rechargements périodiques. Cette turbine de 3,15 MW est exploitée par
I'ENEL et construite par Riva-Hydroart. Il s'agit d'une turbine à axe horizontal, à injecteur unique, fonctionnant sous une chute de $460 \mathrm{~m}$, pour un débit de $0,8 \mathrm{~m}^{3} / \mathrm{s}$ et une vitesse de rotation de $750 \mathrm{tr} / \mathrm{mn}$. La roue, de diamètre extérieur de $1,495 \mathrm{~m}$, comprend dix-huit augets et est moulée en acier $\mathrm{X} 15 \mathrm{Cr} 13$ (Euro Norm).

\section{Erosion}

Avant chaque rechargement de la roue, le volume et la profondeur de la zone érodée ont été relevés par l'ENEL[3]. L'érosion affecte de manière inégale les augets. Ces différences sont probablement dues à leurs géométries différentes suite aux multiples rechargements effectués sans contrôle dimensionnel strict. De plus, les demi-augets droits sont en moyenne deux à trois fois plus érodés que les demi-augets gauches. La zone érodée se. situe en fond d'auget. Elle s'étend, après une année de fonctionnement, sur une surface d'environ $3 \times 2 \mathrm{~cm}^{2}$, avec une profondeur maximale de $7 \mathrm{~mm}$.

\section{Gamme de fonctionnement}

Les conditions normales d'exploitation de la machine varient selon les besoins du réseau et le niveau du réservoir d'alimentation. Elles varient entre 80 et $100 \%$ de la puissance nominale.

\subsection{Essais de marquage}

Les essais de marquage consistent à placer sur la roue rechargée, aux endroits où le maximum d'érosion a été relevé, des échantillons en métal poli miroir épousant parfaitement la forme de l'auget, destinés à enregistrer les indentations produites par l'écoulement cavitant. A la fin de l'essai, les échantillons sont récupérés pour être analysés. 


\begin{tabular}{|c|c|c|c|c|c|c|c|}
\hline Essai & $\mathrm{n}^{\circ}$ auget & Durée (heures) & Hauteur $(\mathrm{m})$ & $\sigma=\mathrm{NPSH} / \mathrm{H}(-)$ & Débit $\left(\mathrm{m}^{3} / \mathrm{s}\right)$ & Puissance $(\mathrm{kWe})$ & $\%$ Puissance \\
\hline p100 & 3 & $2 \mathrm{~h}$ & 432,9 & 0,022 & 0,976 & 3355 & $100 \%$ \\
p90 & 2 & $2 \mathrm{~h} 6 \mathrm{mn}$ & 446,8 & 0,021 & 0,908 & 3057 & $90 \%$ \\
p80 & 18 & $2 \mathrm{~h}$ & 458,6 & 0,021 & 0,804 & 2690 & $80 \%$ \\
\hline
\end{tabular}

Tableau 1. - Conditions d'essais de marquage.

\begin{tabular}{|c|c|c|c|c|c|c|}
\hline Essai & $\mathrm{n}^{\circ}$ auget & demi-auget & $\mathrm{Vd}(\mathrm{pm} / \mathrm{s})$ & $\mathrm{n}\left(\mathrm{nb} / \mathrm{mm}^{2} \cdot \mathrm{s}\right)$ & $\mathrm{hc}(\mu \mathrm{m})$ & $\mathrm{Rc}(\mu \mathrm{m})$ \\
\hline p100 & 3 & droit & 0,38 & 0,00022 & 2,29 & 33 \\
p90 & 2 & droit & 2,54 & 0,00085 & 2,38 & 118 \\
p80 & 18 & droit & 3,72 & 0,00181 & 2,36 & 99 \\
\hline
\end{tabular}

Tableau 2. - Valeurs maximales des paramètres de quantification du marquage.

Remarques: - L'unité utilisée pour le taux volumique de marquage est: $1 \mathrm{pm} / \mathrm{s}=10^{-12} \mathrm{~m} / \mathrm{s}=1 \mu \mathrm{m}^{3} / \mathrm{mm}^{2} . \mathrm{s}$.

- Aucun retrait significatif du produit de moulage n'a pu être mesuré en comparant le marquage sur un échantillon étalon et sur son empreinte.

Lors d'une première campagne d'essais, des échantillons en acier inoxydable 316L (Z3CND17.12 - AFNOR) furent vissés au fond de chaque demi-auget à l'endroit où l'érosion était maximale. Seule la condition à pleine charge fut testée. Aucun marquage significatif ne fut enregistré en quatre heures d'essai. Cette condition de fonctionnement n'était peut-être pas la plus érosive et/ou l'emplacement de l'échantillon ne correspondait pas à la zone d'initiation de l'érosion. Une seconde campagne d'essais fut donc effectuée pour tester d'autres points de fonctionnement de la machine et détecter le marquage sur une plus grande surface. Une autre technique d'enregistrement du marquage fut alors utilisée. Les fonds d'augets érodés sont rechargés par soudage d'acier $316 \mathrm{~L}$ puis meulés au profil initial. Cette surface d'environ $10 \times 8 \mathrm{~cm}^{2}$ est ensuite polie finement afin d'obtenir un poli miroir (cf. fig.1) et de pouvoir enregistrer les impacts. Après l'essai, une empreinte de la surface impactée est prise à l'aide d'un produit de moulage très précis.

\section{Choix des augets}

Pour chacune des conditions d'essai, un auget ayant présenté une forte érosion est sélectionné et poli. Seuls les demiaugets droits présentant une plus forte érosion ont été retenus. Les profondeurs maximales d'érosion sur chacun des trois demi-augets sélectionnés sont données au §4.2.

\section{Conditions d'essais}

Trois essais furent réalisés, balayant les conditions de fonctionnement de la centrale : $100 \%, 90 \%$ et $80 \%$ de la pleine charge. Les conditions d'essais sont données dans le tableau 1.

\subsection{Résultats}

\section{Examen visuel}

Après chaque essai, la surface polie est examinée visuellement. Pour l'essai à pleine charge (p100), très peu d'indentations sont visibles, confirmant les résultats de la première campagne. Pour les deux autres conditions d'essai, un marquage visible est obtenu en deux heures d'essai.

\section{Mesure du marquage par profilométrie}

La surface des empreintes est mesurée à l'aide d'un profilomètre laser (de type UBC14-UBM, d'une précision de $0,06 \mu \mathrm{m}$ en hauteur). Sur chaque empreinte, la zone comportant le plus d'impacts est sélectionnée visuellement et au moins deux surfaces de $4 \times 4 \mathrm{~mm}^{2}$ sont mesurées avec une résolution de 250 points $/ \mathrm{mm}$ (soit une mesure chaque $4 \mu \mathrm{m}$ ). La surface courbe mesurée est ensuite redressée par régression polynomiale de degré 4 , puis analysée à l'aide du logi-

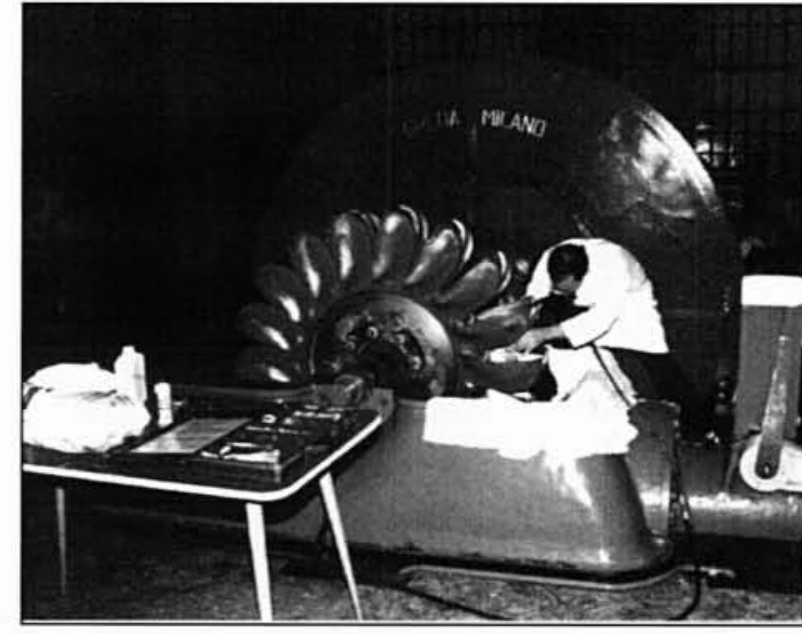

Fig. 1. Polissage sur la roue prototype.

ciel “ ADRESSE ”. Ce logiciel, développé par le LEGI (Laboratoire des Ecoulements Géophysiques et Industriels, Grenoble) dresse la liste de toutes les indentations en calculant leur profondeur, leur volume et leur diamètre.

De cette distribution des impacts, on peut extraire par calcul des valeurs scalaires globales plus faciles d'utilisation et représentatives du marquage subi. Il s'agit des quatre grandeurs suivantes :

( $\mathrm{N}$ est le nombre total d'indentations détectées, $\mathrm{S}$ est l'aire mesurée, $T$ est la durée de l'essai de marquage, $v_{i}, h_{i}$ et $r_{j}$ sont respectivement le volume, la profondeur et le rayon de la i.ème indentation)

- le taux volumique de marquage :

$\mathbf{V d}=\Sigma \mathbf{v}_{i} / \mathbf{S} . \mathbf{T}$

- le taux de marquage :

- la profondeur caractéristique des indentations :

- le rayon caractéristique des indentations :

$\mathrm{n}=\mathrm{N} / \mathrm{S} . \mathrm{T}$

$h c=\Sigma \mathbf{v}_{i} \cdot h_{i} / \Sigma \mathbf{v}_{i}$

$\mathbf{R c}=\Sigma \mathbf{v}_{i} \cdot \mathbf{r}_{i} / \Sigma \mathbf{v}_{i}$

\section{Résultats}

Le tableau 2 donne les valeurs maximales des paramètres de quantification du marquage.

\section{Discussion}

Le marquage enregistré est qualitativement semblable à un marquage obtenu avec une cavitation à poche. La condition d'essai à $80 \%$ de la puissance est le fonctionnement le plus érosif. La condition d'essai à $90 \%$ est d'environ un tiers 


\begin{tabular}{|c|c|c|c|c|c|}
\hline Essai & $\mathrm{n}^{\circ}$ auget & Durée $(6$ jets) (minutes) & Hauteur $(\mathrm{m})$ & $\sigma=\mathrm{NPSH} / \mathrm{H}(-)$ & Débit $(6$ jets $)\left(\mathrm{m}^{3} / \mathrm{s}\right)$ \\
\hline m100 & 11 & $30 \mathrm{mn}$ & 77,10 & 0,023 & 0,201 \\
m-érosion & 11 & $10 \mathrm{mn}$ & 102,07 & 0,018 & 0,246 \\
\hline
\end{tabular}

Tableau 3. - Conditions des essais sur modèle à échelle réduite.

\begin{tabular}{|c|c|c|c|c|c|c|}
\hline Essai & $\mathrm{n}^{\circ}$ auget & demi-auget & $\mathrm{Vd}(\mathrm{pm} / \mathrm{s})$ & $\mathrm{n}\left(\mathrm{n} / \mathrm{mm}^{2} \cdot \mathrm{s}\right)$ & $\mathrm{hc}(\mu \mathrm{m})$ & $\mathrm{Rc}(\mu \mathrm{m})$ \\
\hline $\mathrm{m} 100$ & 11 & gauche & 10,97 & 0,0023 & 2,05 & 124 \\
" & & droit & 33,41 & 0,0140 & 4,68 & 115 \\
m-érosion & \multirow{2}{*}{11} & gauche & 14,17 & 0,0096 & 1,98 & 56 \\
", & & droit & 36,76 & 0,0118 & 2,58 & 84 \\
\hline
\end{tabular}

Tableau 4. - Paramètres de quantification du marquage.

moins érosive. Pour ces deux conditions, les caractéristiques géométriques des indentations (hc et $\mathrm{Rc}$ ) sont du même ordre de grandeur : un peu plus de $2 \mu \mathrm{m}$ de profondeur pour un rayon d'environ $0,1 \mathrm{~mm}$. La condition d'essai à pleine charge est la moins érosive (le taux de marquage volumique est environ dix fois moindre qu'à $80 \%$ ). La profondeur caractéristique des indentations est du même ordre de grandeur qu'à $80 \%$ et $90 \%$ mais le rayon caractéristique est trois fois moindre.

Les essais de marquage ont permis d'identifier et de quantifier les fonctionnements érosifs de la machine. Ils apportent ainsi une information précieuse à l'exploitant pour optimiser la conduite de l'installation vis-à-vis de l'érosion et pour prévoir plus précisément les périodes de rechargement.

\section{III — ESSAIS SUR LE MODÈLE}

\subsection{Plate-forme d'essais et échantillons de marquage}

Les essais sur modèle à échelle réduite se sont déroulés sur la plate-forme d'essais de turbines Pelton du constructeur Riva-Hydroart. Cette plate-forme d'essai permet la mise en dépression de la cuve avale à surface libre (pression d'air jusqu'à 0,16 atmosphère) afin de respecter la similitude en pression et atteindre des régimes cavitants. La roue est montée sur un axe vertical et alimentée par six injecteurs. La vitesse de rotation est de $1100 \mathrm{tr} / \mathrm{mn}$.

La roue modèle est composée de dix-huit augets démontables vissés sur le moyeu, en exacte similitude géométrique avec la roue prototype (rapport d'échelle de 3,5). Les augets sont moulés par Riva-Hydroart en aluminium AlMg5 (AG3T - AFNOR, recuit) et sont la réplique de l'auget prototype $n^{\circ} 11$. Cet auget présente une érosion représentative de la roue, avec une plus forte érosion sur le demi-auget droit. La surface intérieure des augets est polie miroir.

\subsection{Conditions d'essais}

Deux conditions d'essais furent réalisées :

- une condition, notée "m100", en similitude avec la première condition testée sur le prototype (p100),

- une condition, notée "m-érosion", correspondant à la chute maximale possible sur la plate-forme d'essais, et pour laquelle des essais de perte de masse avaient été réalisés auparavant par Riva-Hydroart.

Les essais sur le modèle se sont déroulés avant la deuxième campagne d'essais sur le prototype. C'est pourquoi seule la condition à pleine charge a été testée sur le modèle car elle était supposée être la plus érosive.

L'obtention des conditions d'écoulement stabilisées est réalisée de manière à réduire au minimum la durée des écoulements transitoires. Le bassin aval est mis en dépression afin d'opérer au NPSH désiré, la roue étant immobile. Puis les injecteurs sont ouverts jusqu'à la course voulue et la vitesse de rotation est atteinte en moins de trois minutes. A la fin de l'essai, la roue est arrêtée en moins de quarante secondes.

\section{Similitude}

Le rapport d'échelle géométrique entre le prototype et le modèle est de :

$$
\lambda=\mathrm{Lp} / \mathrm{Lm}=3,5 .
$$

Le rapport de vitesse de rotation entre le prototype et le modèle est de :

$$
\mathrm{K}=\mathrm{Np} / \mathrm{Nm}=750 / 1100=0,68 \text {. }
$$

D'après les lois de similitude pour les machines hydrauliques, les grandeurs hydrauliques du prototype se déduisent de celles du modèle par les relations suivantes :

- pour les vitesses fluides :

$$
\mathrm{Cp} / \mathrm{Cm}=\lambda \mathrm{K}=2,4,
$$

- pour les débits :

$$
\mathrm{Qp} / \mathrm{Qm}=\lambda^{3} \mathrm{~K}=29,2,
$$

- pour les hauteurs :

$$
\mathrm{Hp} / \mathrm{Hm}=\lambda^{2} \mathrm{~K}^{2}=5,7 \text {. }
$$

Pour respecter la similitude en pression, les essais sont effectués pour $\sigma=\mathrm{NPSH} / \mathrm{H}$ constant, où le NPSH correspond à la pression dans la cuve avale et où $\mathrm{H}$ est la hauteur.

\subsection{Résultats}

Un marquage visible a été enregistré sur le modèle, en fond d'auget comme sur le prototype, pour les deux conditions d'essais en des temps d'essais courts (30 mn et $10 \mathrm{mn}$ ). La méthode de mesure du marquage est la même que pour les essais sur le prototype. Les paramètres de quantification du marquage sont présentés dans le tableau 4 (la durée d'essai $\mathrm{T}$ utilisée pour le calcul de $\mathrm{Vd}$ est la durée réelle des essais : $30 \mathrm{mn}$ et $10 \mathrm{mn}$ ).

\section{Discussion}

La condition " m-érosion" est légèrement plus érosive que la condition " m100" (rapport entre 1,1 et 1,4 pour le taux volumique de marquage). L'écoulement sur le demi-auget droit est deux à trois fois plus érosif que sur le demi-auget gauche et les dimensions caractéristiques des indentations sont également plus grandes sur le demi-auget droit. Cette différence de marquage entre les deux demi-augets est correctement corrélée avec les pertes de masse relevées sur le prototype. 
- Pour Vd

\begin{tabular}{|c|c|c|cc|}
\hline Essais modèle & Vd modèle $(\mathrm{pm} / \mathrm{s})$ & Vd proto transposé $(\mathrm{pm} / \mathrm{s})$ & \multicolumn{2}{c|}{ Vd proto mesuré $(\mathrm{pm} / \mathrm{s})$} \\
\hline m100 & 5,56 & 2,16 & $\mathrm{p} 100:$ & 0,38 \\
m-érosion & 6,12 & 1,71 & p90: & 2,54 \\
& & & p80: & 3,72 \\
\hline
\end{tabular}

- pour hc:

\begin{tabular}{|c|c|c|cc|}
\hline Essais modèle & hc modèle $(\mu \mathrm{m})$ & hc proto transposé $(\mu \mathrm{m})$ & \multicolumn{2}{c|}{ hc proto mesuré $(\mu \mathrm{m})$} \\
\hline m100 & 4,68 & 1,58 & p100: & 2,29 \\
m-érosion & 2,58 & 0,94 & p90: & 2,38 \\
& & & p80: & 2,36 \\
\hline
\end{tabular}

- pour Rc:

\begin{tabular}{|c|c|c|cc|}
\hline Essais modèle & Rc modèle $(\mu \mathrm{m})$ & Rc proto transposé $(\mu \mathrm{m})$ & \multicolumn{2}{c|}{ Rc proto mesuré $(\mu \mathrm{m})$} \\
\hline m100 & 115 & 388 & p100: & 36 \\
m-érosion & 84 & 253 & p90: & 118 \\
& & & p80: & 99 \\
\hline
\end{tabular}

Tableau 5. - Valeurs transposées, comparées aux grandeurs mesurées sur le prototype.

Remarque : pour le modèle, la valeur du taux volumique de marquage a été rapportée à un injecteur, qui est la configuration du prototype.

\subsection{Essai de perte de masse}

Un essai de vingt quatre heures, effectué dans la condition " m-érosion", a permis d'obtenir une forte érosion sur le modèle. Une érosion d'environ $2 \mathrm{~mm}$ de profondeur a été obtenue sur le demi-auget droit, tandis qu'une érosion légère (moins de $0,2 \mathrm{~mm}$ de profondeur) a été relevée sur le demiauget gauche (cf. fig.2). La zone érodée sur le demi-auget droit a été mesurée à l'aide d'un profilomètre laser. Le volume érodé est de $51,35 \mathrm{~mm}^{3}\left( \pm 0,05 \mathrm{~mm}^{3}\right)$.

Ces essais modèles réalisés en respectant la similitude en pression ont donc permis :

- de mettre en évidence sur le modèle un marquage par cavitation (l'eau n'étant pas chargée en particules solides, il ne peut s'agir d'érosion par abrasion),

- de retrouver un comportement de l'érosion semblable à celui constaté sur le prototype (emplacement de la zone érodée, dissymétrie entre les demi-augets),

- d'effectuer un essai de perte de masse en une durée raisonnable.

Le constructeur peut donc déterminer à partir d'essais sur modèle si une géométrie est susceptible d'érosion ou non, et donc modifier son dessin avant la fabrication de la roue prototype.

\section{IV a APPLICATION D’UNE MÉTHODE DE PRÉVI- SION DE L'ÉROSION}

La méthode de prévision de l'érosion utilisée est dans ses grandes lignes celle proposée par Lecoffre et al. [4][5]. Cette prévision est basée sur le concept d'agressivité de l'écoulement. L'agressivité est le chargement mécanique d'origine hydrodynamique subi par le métal de l'auget, c'est-à-dire l'ensemble des implosions générées par l'écoulement. Le marquage, qui est le premier signe de l'endommagement mécanique du matériau par l'écoulement cavitant, est la manifestation de l'agressivité.

La prévision se fait en deux temps :

- dans un premier temps, l'agressivité, est enregistrée, quantifiée puis transposée du modèle au prototype, en tenant compte de la différence d'échelle, de vitesse et de matériau,

- dans un deuxième temps, des corrélations expérimentales,

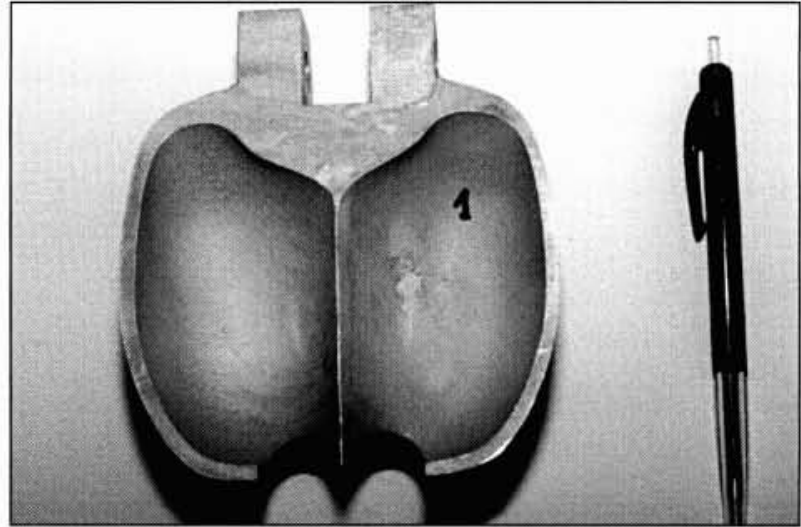

Fig. 2. Auget modèle érodé.

obtenues par des essais en laboratoire sur le matériau concerné, permettent de déduire la profondeur de l'érosion à partir de l'agressivité.

\section{- 4.1. Transposition de l'agressivité du modèle au prototype}

Seule la condition à pleine charge a été testée à la fois sur le modèle et sur le prototype. Cette condition n'est cependant pas la plus agressive sur le prototype. Néanmoins, les grandeurs de l'agressivité sont transposées du modèle au prototype, afin de comparer les ordres de grandeur. Des essais de marquage complémentaires sur le modèle sont prévus courant 1997 pour tester les conditions à $90 \%$ et $80 \%$ de la pleine charge.

\section{Description}

La méthode de transposition de l'agressivité a été développée par l'INPG/LEGI en collaboration avec EDF à partir d'un modèle numérique de déformation d'un matériau élastoplastique soumis à une onde de surpression sphérique [6][7]. Le matériau est caractérisé principalement par deux paramètres : son module d'Young et sa contrainte limite en cisaillement. Le modèle permet de déduire de la géométrie d'une indentation (profondeur h, rayon r) l'onde de surpression qui en est 
la cause (caractérisée par la pression maximale en paroi $\mathrm{P}$, et la distance du centre d'émission de l'onde à la paroi L). On peut donc calculer le chargement en ondes de pression à partir de la géométrie des trous mesurée sur l'échantillon et réciproquement, on peut calculer la géométrie des trous que produirait un chargement en ondes de pression donné.

Le marquage mesuré sur les augets modèle en aluminium est transposé à un marquage sur les augets prototype en acier $316 \mathrm{~L}$ par l'intermédiaire du chargement en ondes de pression.

1- Le marquage mesuré sur l'auget modèle en aluminium donne le chargement.

2- Ce chargement en ondes de pression (P, L) est ensuite transposé en échelle et en vitesse. Dans ce modèle :

- la distance des ondes à la paroi est proportionnelle à

l'échelle géométrique, donc $\mathrm{L}$ est multiplié par $\lambda$.

- la pression en paroi est proportionnelle à la vitesse de l'écoulement, donc $\mathrm{P}$ est multiplié par $\lambda \mathrm{K}$.

3- Ce chargement est appliqué au matériau de l'auget prototype en acier $316 \mathrm{~L}$ et produit le marquage sur le prototype, dont les grandeurs représentatives $\mathrm{Vd}$, hc et Rc sont extraites. Cette méthode effectue la transposition pour chaque indentation, avant de déduire les valeurs représentatives de l'agressivité : Vd, hc et Rc.

\section{Application}

Les valeurs transposées, comparées aux grandeurs mesurées sur le prototype, sont données dans le tableau 5.

Cette méthode de transposition de l'agressivité donne un assez bon ordre de grandeur pour le taux volumique de marquage, qui est le paramètre principal de l'agressivité, mais les dimensions caractéristiques des indentations sont mal estimées : hc est sous-estimé alors que Rc est surestimé. Les essais complémentaires sur le modèle permettront une évaluation plus précise de la méthode.

\subsection{Prévision de l'érosion à partir de l'agressivité mesurée sur le prototype}

Le CERG dispose d'un appareil de laboratoire - le CAVERSIM - permettant de reproduire de façon accélérée un chargement cavitant sur tous types de matériaux par implosion d'un tourbillon de vapeur. Pour l'acier $316 \mathrm{~L}$ et pour l'acier constitutif de la turbine, le CERG a procédé à des essais de marquage et d'érosion en enregistrant la profondeur de l'érosion en fonction du temps [8]. Connaissant l'agressivité, la méthode utilisée permet de déduire la profondeur de l'érosion du prototype par une simple transposition en temps, à partir des essais de référence sur le CAVERSIM [9].
Conversion de l'agressivité du 316L au matériau constitutif de la roue

Le matériau utilisé pour les essais de marquage (316L) n'est pas le matériau constitutif de la roue (X15Cr13) qui est soumis à l'érosion. Afin de prédire l'érosion sur la roue, le marquage mesuré sur le $316 \mathrm{~L}$ est converti en un marquage sur l'acier " roue ". Comme les caractéristiques de cet acier ne sont pas connues, la méthode de transposition employée précédemment ne peut s'appliquer. De manière plus grossière, on comparera à des essais de marquage de référence effectués par le CERG à l'aide du CAVERSIM sur ces deux matériaux. L'hypothèse d'une même proportionnalité, pour les deux matériaux, entre le marquage sur le prototype et le marquage du CAVERSIM permet de déduire le marquage cherché :

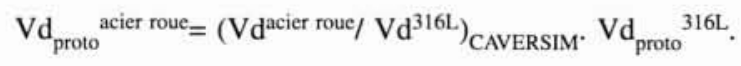

Mesures de référence:

\begin{tabular}{|c|c|c|}
\hline CAVERSIM & $\begin{array}{c}\text { Vd - acier 316L } \\
(\mathrm{pm} / \mathrm{s})\end{array}$ & $\begin{array}{c}\text { Vd - acier "roue" } \\
(\mathrm{pm} / \mathrm{s})\end{array}$ \\
\hline condition TC2 & 1935 & 760 \\
\hline
\end{tabular}

Conversion:

\begin{tabular}{|c|c|c|}
\hline Pelton prototype & $\begin{array}{c}\mathrm{Vd}-\text { acier 316L } \\
(\mathrm{pm} / \mathrm{s})\end{array}$ & $\begin{array}{c}\mathrm{Vd} \text { - acier "roue" } \\
\text { déduit }(\mathrm{pm} / \mathrm{s})\end{array}$ \\
\hline condition p100 & 0,38 & 0,15 \\
condition p90 & 2,54 & 1,00 \\
condition p80 & 3,72 & 1,46 \\
\hline
\end{tabular}

\section{Calcul de la profondeur d'érosion}

La profondeur d'érosion $\mathrm{Z}$ sur la machine après un temps $\mathrm{T}$ est donnée par la profondeur d'érosion relevée sur le CAVERSIM sur le même matériau, en appliquant une simple transposition en temps. Le coefficient multiplicateur appliqué au temps est égal au rapport de l'agressivité du CAVERSIM à celle du prototype.

Soit :

$$
\mathrm{Z}(\mathrm{T})=\mathrm{Z}_{\mathrm{CAV}}\left(\mathrm{T}_{\mathrm{CAV}}\right) \text {, avec } \mathrm{T}=\mathrm{T}_{\mathrm{CAV}} *\left(\mathrm{Vd}_{\mathrm{CAV}} / \mathrm{Vd}\right) \text {. }
$$

Les résultats pour les trois conditions d'essais sont rassemblés dans le tableau 6.

\section{Erosions mesurées sur le prototype}

Dans le tableau 7 sont indiquées les profondeurs maximales d'érosion (Z) mesurées sur chacun des trois demi-augets sélectionnés, relevées lors de trois rechargements successifs.

\begin{tabular}{|c|c|c|c|c|}
\hline \multicolumn{2}{|c|}{ CAVERSIM } & \multicolumn{3}{|c|}{ Prototype } \\
\hline Condition & $\mathrm{TC} 2$ & $\mathrm{p} 100$ & $\mathrm{p} 90$ & $\mathrm{p} 80$ \\
\hline $\mathrm{T}_{\mathrm{CAV}}(\mathrm{h})$ & $\mathrm{Z}_{\mathrm{CAV}}=\mathrm{Z}_{\text {proto }}(\mathrm{mm})$ & $\mathrm{T}_{\text {proto }}(\mathrm{h})$ & $\mathrm{T}_{\text {proto }}(\mathrm{h})$ & $\mathrm{T}_{\text {proto }}(\mathrm{h})$ \\
10 & 0,00 & 50000 & 7600 & 5190 \\
20 & 0,13 & 100000 & 15200 & 10380 \\
30 & 0,19 & 150000 & 22800 & 15570 \\
40 & 0,25 & 200000 & 30400 & 20760 \\
\hline
\end{tabular}

Tableau 6. - Profondeur d'érosion pour les diverses conditions d'essais.

\begin{tabular}{|c|c|c|c|}
\hline $\mathrm{n}^{\circ}$ auget & relevé $\mathrm{n}^{\circ} 1: 5796$ heures & relevé $\mathrm{n}^{\circ} 2: 3604$ heures & relevé $\mathrm{n}^{\circ} 3: 4589$ heures \\
\hline 3 droit & $\mathrm{Z}=3,1 \mathrm{~mm}$ & $\mathrm{Z}=3,8 \mathrm{~mm}$ & $\mathrm{Z}=5,0 \mathrm{~mm}$ \\
2 droit & $\mathrm{Z}=2,4 \mathrm{~mm}$ & $\mathrm{Z}=2,5 \mathrm{~mm}$ & $\mathrm{Z}=2,7 \mathrm{~mm}$ \\
18 droit & $\mathrm{Z}=3,7 \mathrm{~mm}$ & $\mathrm{Z}=3,1 \mathrm{~mm}$ & $\mathrm{Z}=3,3 \mathrm{~mm}$ \\
\hline
\end{tabular}

Tableau 7. - Profondeurs maximales d'érosion. 


\section{Discussion}

En considérant un fonctionnement continu à la condition la plus érosive ( $\mathrm{p} 80$ ), la méthode de prévision donne une érosion de $0,1 \mathrm{~mm}$ au bout de 8000 heures. L'érosion réelle mesurée sur le prototype étant d'environ $3 \mathrm{~mm}$ pour 5000 heures de fonctionnement entre $100 \%$ et $80 \%$ de la puissance nominale, les érosions prédites par la méthode sont largement inférieures aux érosions mesurées.

Cette méthode de prévision de la profondeur d'érosion par une simple transposition en temps des mesures du CAVERSIM suppose que le mode de chargement est le même sur le CAVERSIM et sur la Pelton. Or, les indentations produites par le CAVERSIM sur le $316 \mathrm{~L}$ sont de dimensions plus faibles $(\mathrm{hc}=1 \mu \mathrm{m}$ et $\mathrm{Rc}=61 \mu \mathrm{m})$ que celles mesurées sur le prototype (hc d'environ $2 \mu \mathrm{m}$ et Rc d'environ $100 \mu \mathrm{m}$ ). L'érosion produite par le CAVERSIM est donc moins sévère, du point de vue de la taille des impacts, que celle sur le prototype.

De plus, l'altération du profil de l'auget par l'érosion naissante accélère sans doute le phénomène d'érosion sur la turbine. En effet, l'expérience du constructeur montre que de très faibles écarts de géométrie (de l'ordre du dixième de millimètre) influencent considérablement le comportement en cavitation. L'érosion agit en retour sur l'écoulement en le rendant plus érosif, cet effet de rétroaction n'étant probablement pas sensible pour le CAVERSIM.

\section{$\mathrm{V} \square$ CONCLUSION}

Les essais de marquage réalisés sur la turbine Pelton prototype pour les trois conditions courantes de fonctionnement ont permis d'identifier et de quantifier les fonctionnements érosifs. Sur modèle, les essais effectués en respectant la similitude en pression ont également révélé un marquage de cavitation en fond d'auget. De plus, un essai de perte de masse de relativement courte durée a permis d'obtenir une érosion importante, semblable à celle constatée sur le prototype. Une méthode de prévision de l'érosion sur le prototype à partir de la mesure de l'agressivité sur le modèle a été appliquée. Cette méthode, si elle permet d'évaluer l'ordre de grandeur de l'agressivité transposée du modèle au prototype, sous-estime l'érosion produite.

Cette mauvaise estimation est sans doute liée à une distribution d'impacts de cavitation notablement différente de celle produite par le moyen de laboratoire utilisé pour établir la corrélation agressivité/perte de masse. La cavitation dans les turbines Pelton reste pour une large part méconnue quant à sa forme et à ses conditions d'apparition. Le phénomène semble extrêmement sensible à la géométrie des augets. Les essais sur le modèle ont montré que, à défaut d'en connaître l'origine, le constructeur pouvait déterminer si une géométrie était susceptible d'érosion ou non et donc modifier son dessin avant la fabrication du prototype.

\section{REMERCIEMENTS}

Ces essais se sont déroulés grâce au support financier du programme BRITE-EURAM de la Communauté Européenne. Les auteurs remercient également les partenaires du programme pour le travail constructif réalisé en commun et pour leur autorisation de publication.

\section{RÉFÉRENCES}

[1] Grein (1990) " Cavitation pitting and rain erosion on Pelton runners" AIRH Symp., Belgrade.

[2] BRIVIO, ZAPPI (1995) "La cavitazione nelle turbine Pelton”, L'Energia Elettrica, Vol.72, Mars-Avril 1995.

[3] ENEL, (1994), “ CETO-PALOBBIA: evaluation of surfaces damaged by cavitation ", rapports ENEL.

[4] Lecoffre, Marcoz, Franc, Michel, (1985) " Tentative procedure for scaling cavitation damage " ASME Int. Symp. on Cavitation in Hydraulic Structure and Turbomachinery, Albuquerque.

[5] LeCOFFre, (1995) “ Cavitation erosion, hydrodynamic scaling laws, practical method of long term damage prediction ", Int. Symp. on Cavitation CAV'95, Deauville.

[6] Reboud (1987) " Réponse impulsionnelle d'un milieu élastoplastique: application à l'étude de l'érosion de cavitation ", Thèse de Doctorat (Mécanique), INPG, Grenoble.

[7] Fortes Patella (1994) “ Analyse de l'érosion de cavitation par simulation numérique d'impacts ", Thèse de Doctorat (Mécanique), INPG, Grenoble.

[8] CERG, BRITE-EURAM PROJECT (1995), “ Erosion measurements on CAVERSIM ", rapport CERG.

[9] Lavigne, Retailleau, Woillez (1995) “ Measurement of the aggressivity of erosive cavitating flows by a technique of pits analysis. Application to a method of prediction of erosion ", Int. Symp. on Cavitation, CAV'95, Deauville. 